\title{
SMALL DISCS CONTAINING CONJUGATE ALGEBRAIC INTEGERS
}

\author{
Artūras Dubickas \\ Vilnius University, Faculty of Mathematics and Informatics, Institute of Mathematics \\ Naugarduko 24, LT-03225 Vilnius, Lithuania; arturas.dubickas@mif.vu.lt
}

\begin{abstract}
In this note we show that, for any $\xi \in \mathbf{R}$, there is an infinite set of positive integers $S$ such that, for each $d \in S$, the open disc with center at $\xi$ and radius $1+(\log \log d)^{2} /(2 \log d)$ contains a full set of conjugates of an algebraic integer of degree $d$. A slightly better bound on the radius is established when $\xi \in \mathbf{Q} \backslash \mathbf{Z}$.
\end{abstract}

\section{Introduction}

For $E \subseteq \mathbf{C}$, the quantity

$$
\tau(E):=\lim _{n \rightarrow \infty} \sup _{z_{1}, \ldots, z_{n} \in E}\left(\prod_{1 \leq i<j \leq n}\left|z_{i}-z_{j}\right|\right)^{2 / n(n-1)}
$$

is called the transfinite diameter (or logarithmic capacity) of $E$. It is known that a (closed or open) disc with radius $R$ has transfinite diameter $R$, whereas an interval of lenght $I$ has transfinite diameter $I / 4$. In [7], Fekete has shown that every compact set $E$ satisfying $\tau(E)<1$ contains only finitely many full sets of conjugate algebraic integers over $\mathbf{Q}$. In particular, this result can be applied to every closed disc whose radius is smaller than 1 and to every real interval whose length is smaller than 4 .

In the opposite direction, Fekete and Szegö [8] proved that if $E$ is a compact set which is stable under complex conjugation and satisfies $\tau(E) \geq 1$, then its every complex neighborhood $F$ (so that $E \subset F$ and $F$ is an open set) contains infinitely many sets of conjugate algebraic integers. Furthermore, by the results of Robinson [15] and Ennola [4], every real interval of length strictly greater than 4 also contains infinitely many sets of conjugate algebraic integers.

In [18], Zaïmi gave a lower bound for the length of a real interval containing an algebraic integer of degree $d$ and its conjugates. His result asserts that the length $I$ of such an interval should be at least $4-\phi(d)$, where $\phi(d)$ is some explicit positive function which tends to zero as $d \rightarrow \infty$. (For instance, one can take $\phi(d)=(c \log d) / d$ with some $c>0$. Similar bound also follows from an earlier result of Schur [17].) On the other hand, the author has shown that, for infinitely many $d \in \mathbf{N}$, every real interval of length $4+4(\log \log d)^{2} / \log d$ contains an algebraic integer of degree $d$ and its conjugates (see [2] and [3]). It is not known whether there is an interval $[t, t+4]$ with some $t \in \mathbf{R} \backslash \mathbf{Z}$ containing infinitely many full sets of algebraic integers. For $t \in$ $\mathbf{Z}$, one can simply take infinitely many algebraic integers of the form $t+2 \cos (\pi r)+2$, where $r \in \mathbf{Q}$. By Kronecker's theorem [13], these are the only such numbers in $[t, t+4]$ if $t \in \mathbf{Z}$.

https://doi.org/10.5186/aasfm.2020.4524

2010 Mathematics Subject Classification: Primary 11R04, 11R09.

Key words: Conjugate algebraic integers, disc with real center, diameter of algebraic integer, Rouché's theorem. 
In this note, we shall consider discs with real centers and radii close 1. Suppose first that an algebraic integer $\alpha$ of degree $d$ lies with its conjugates in the disc $|z-\xi| \leq$ $R_{d}$, where $\xi \in \mathbf{R}$ and $R_{d}>0$. Then, we can write the discriminant

$$
D=\prod_{1 \leq i<j \leq d}\left(\alpha_{i}-\alpha_{j}\right)^{2}=\prod_{1 \leq i<j \leq d}\left(\left(\alpha_{i}-\xi\right)-\left(\alpha_{j}-\xi\right)\right)^{2}
$$

of $\alpha$ with conjugates $\alpha_{1}=\alpha, \alpha_{2}, \ldots, \alpha_{d}$ as the square of Vandermonde determinant with rows $\left(\alpha_{1}-\xi\right)^{j-1}, \ldots,\left(\alpha_{d}-\xi\right)^{j-1}$, where $j=1, \ldots, d$. Using the upper bound $\sum_{i=1}^{d}\left|\alpha_{i}-\xi\right|^{2(j-1)} \leq d R_{d}^{2 j-2}$, by Hadamard's inequality, we obtain

$$
|D| \leq \prod_{j=1}^{d} d R_{d}^{2 j-2}=d^{d} R_{d}^{d(d-1)} .
$$

This yields $R_{d} \geq\left|D_{d}\right|^{1 / d(d-1)} d^{-1 /(d-1)}$, where $\left|D_{d}\right|$ stands for the smallest discriminant of an algebraic number field of degree $d$. From $\left|D_{d}\right|>1$ it follows that

$$
R_{d}>d^{-1 /(d-1)}
$$

for $d \geq 2$. Moreover, $\left|D_{d}\right|>22^{d}$ for $d$ large enough (see [14]). Hence,

$$
R_{d}>1-\frac{\log d}{d}
$$

for $d$ large enough. In the opposite direction we prove the following:

Theorem 1. For any real number $\xi$, there is an infinite set of positive integers $S$ such that, for each $d \in S$, the open disc with center at $\xi$ and radius $1+(\log \log d)^{2} /(2 \log d)$ contains a full set of conjugates of an algebraic integer of degree $d$.

Recall that the diameter of an algebraic integer $\alpha$ of degree $d$ with conjugates $\alpha_{i}$, $i=1, \ldots, d$, is defined by $\max _{1 \leq i<j \leq d}\left|\alpha_{i}-\alpha_{j}\right|$. In this context, Theorem 1 implies that the diameter of the algebraic integer of degree $d$ whose existence is claimed in the theorem is less than $2+(\log \log d)^{2} / \log d$.

It is clear that the diameter of a root of unity shifted by an integer, namely, $e^{2 \pi i / n}+t$, where $t \in \mathbf{Z}$ and $n \in \mathbf{N}$, of degree $d=\varphi(n)$ is less than or equal to 1 . In [10], Grandcolas computed the smallest possible diameters of algebraic integers of degree $d$ up to 10. These computations show that, for each $d$ from 2 to 10 except for $d=9$, there is an algebraic integer, other than $e^{2 \pi i / n}+t$, whose diameter is less than 2. Apparently, the are no such numbers of degree $d \geq 11$, but this is very far from being proved. If proved, this would imply that if an algebraic integer $\alpha$ of degree $d$ is not a shifted root of unity and lies with its conjugates in a closed disc with radius $R_{d}$, then instead of (1) the stronger inequality $R_{d}>1$ holds for $d \geq 11$. Some related results can be found in [11], [12]. See also [1], [9] for the calculations of small diameters of totally real algebraic integers and [5], [6] for the constructions of conjugate algebraic numbers lying on a circle $|z-\xi|=R$.

The main ingredient in the proof of Theorem 1 is its version for rational $\xi$ with a slightly better estimate in $d$.

Theorem 2. Let $p \neq 0$ and $q \geq 2$ be two coprime integers. Then, there is an infinite set of positive integers $S$ such that, for each $d \in S$, the open disc with center at $p / q$ and radius

$$
1+\frac{\log (3 q) \log \log d}{\log d}
$$


contains a full set of conjugates of an algebraic integer of degree $d$.

Note that in case $p=0$ or $q=1$, we have $t=p / q \in \mathbf{Z}$. Then, as we already remarked above, for any $n \in \mathbf{N}$, there is is an algebraic integer $\alpha=e^{2 \pi i / n}+t$ of degree $d=\varphi(n)$ lying with its conjugates on the circle with center at $t$ and radius 1 .

In the next section we prove Theorem 2. Then, in Section 3 we will prove Theorem 1.

\section{Proof of Theorem 2}

Fix two positive integers $K<d$ and write

$$
\left(x-\frac{p}{q}\right)^{d}=x^{d}+\sum_{k=1}^{K}(-1)^{k}\left(\begin{array}{l}
d \\
k
\end{array}\right)\left(\frac{p}{q}\right)^{k} x^{d-k}+\sum_{k=0}^{d-K-1}(-1)^{d-k}\left(\begin{array}{l}
d \\
k
\end{array}\right)\left(\frac{p}{q}\right)^{d-k} x^{k} .
$$

Let $D_{K}$ be the least common multiple of $1,2, \ldots, K$. For each $k \in\{1, \ldots, K\}$, the coefficient

$$
a_{d-k}:=(-1)^{k}\left(\begin{array}{l}
d \\
k
\end{array}\right)\left(\frac{p}{q}\right)^{k}=(-1)^{k} \frac{d}{k q^{K}}\left(\begin{array}{l}
d-1 \\
k-1
\end{array}\right) p^{k} q^{K-k}
$$

is an even integer if

$$
2 D_{K} q^{K} \text { divides } d \text {. }
$$

The proof of the theorem consists in the construction of the polynomial of the form

$$
\begin{aligned}
f(x) & =\left(x-\frac{p}{q}\right)^{d}+\sum_{k=0}^{d-K-1} b_{k}\left(x-\frac{p}{q}\right)^{k} \\
& =x^{d}+a_{d-1} x^{d-1}+\cdots+a_{d-K} x^{d-K}+\sum_{k=0}^{d-K-1} a_{k} x^{k}
\end{aligned}
$$

with some specially chosen $b_{0}, \ldots, b_{d-K-1} \in \mathbf{Q}$. Observe that $a_{d-k}$ are as in (2) for $k=1, \ldots, K$. Also, for each $k$ in the range $0 \leq k \leq d-K-1$, one has

$$
a_{k}=b_{k}+(-1)^{d-k}\left(\begin{array}{l}
d \\
k
\end{array}\right)\left(\frac{p}{q}\right)^{d-k}+\sum_{j=k+1}^{d-K-1}(-1)^{j-k} b_{j}\left(\begin{array}{l}
j \\
k
\end{array}\right)\left(\frac{p}{q}\right)^{j-k} .
$$

Thus, step by step, we can first choose $b_{d-K-1} \in \mathbf{Q}$, then $b_{d-K-2} \in \mathbf{Q}$, etc. up to $b_{0} \in \mathbf{Q}$ so that the coefficients $a_{d-K-1}, \ldots, a_{0}$ are all integers. Furthermore, iteratively we can select $b_{d-K-1}, \ldots, b_{1} \in(-1,1] \cap \mathbf{Q}$ so that the integers $a_{d-K-1}, \ldots, a_{1}$ all even, and after that select $b_{0} \in(-2,2] \cap \mathbf{Q}$ so that that the integer $a_{0}$ is 2 modulo 4 . With this choice, by Eisenstein's criterion with respect to the prime 2, the above monic polynomial $f(x) \in \mathbf{Z}[x]$ of degree $d$ will be irreducible over $\mathbf{Q}$ provided (3) holds.

Let us consider $d$ of the form $d=2 D_{K} q^{K}$, so that (3) surely holds. Fix a small positive number $\delta$. Then, by the Prime Number Theorem, for each $K \geq K(\delta)$, we have $\log D_{K}<(1+\delta) K$ (see, e.g., [16]), and hence

$$
\log d=\log \left(2 D_{K}\right)+K \log q<\log 2+(1+\delta+\log q) K<K \log (2.8 q) .
$$

Accordingly,

$$
K>\frac{\log d}{\log (2.8 q)}
$$


By Rouché's theorem, the polynomials $f(x)$ and $(x-p / q)^{d}$ have the same number of roots in the open disc

$$
\left|x-\frac{p}{q}\right|<R:=1+\frac{\log (3 q) \log \log d}{\log d}
$$

(i.e., they both have $d$ roots) if, for their difference

$$
\varphi(x)=f(x)-\left(x-\frac{p}{q}\right)^{d}=\sum_{k=0}^{d-K-1} b_{k}\left(x-\frac{p}{q}\right)^{k},
$$

the inequality $|\varphi(x)|<\left|(x-p / q)^{d}\right|=R^{d}$ is true for every $x \in \mathbf{C}$ on the circle $|x-p / q|=R$. Then, $f(x) \in \mathbf{Z}[x]$ is an irreducible monic polynomial of degree $d$ with all $d$ roots in $|x-p / q|<R$, and so it defines an algebraic integer of degree $d=2 D_{K} q^{K}$ with required properties.

Since $\left|b_{0}\right| \leq 2$ and $\left|b_{1}\right|, \ldots,\left|b_{d-K-1}\right| \leq 1$, it remains to verify that

$$
2+\sum_{k=1}^{d-K-1} R^{k}<R^{d}
$$

Notice that (5) is equivalent to $R^{d-K}-1<(R-1)\left(R^{d}-1\right)$. Multiplying by $R^{K-d}$ we obtain $1-R^{K-d}<R^{K}(R-1)\left(1-R^{-d}\right)$. This is clearly true if $R^{K}(R-1) \geq 1$. By (4), for $K$ large enough (and so $d$ large enough), we deduce that

$$
R^{K}>\left(1+\frac{\log (3 q) \log \log d}{\log d}\right)^{\log d / \log (2.8 q)}>e^{\log \log d}=\log d .
$$

Hence, $R^{K}(R-1)>\log (3 q) \log \log d>1$, as claimed. This implies (5).

\section{Proof of Theorem 1}

There is nothing to prove if $\xi \in \mathbf{Z}$. We can simply take the disc with radius 1 . If $\xi=p / q$ with coprime integers $p \neq 0$ and $q \geq 2$, then the result follows by Theorem 2 .

From now on, we assume that $\xi$ is irrational. Then, by Dirichlet's theorem, there is an infinite sequence of positive integers $q_{1}<q_{2}<q_{3}<\ldots$ such that, for every $n \in \mathbf{N}$,

$$
\left|\xi-\frac{p_{n}}{q_{n}}\right|<\frac{1}{q_{n}^{2}}
$$

with some $p_{n} \in \mathbf{Z}$. For each $n \in \mathbf{N}$, we select

$$
K_{n}:=q_{n}^{2} \quad \text { and } \quad d_{n}:=2 D_{K_{n}} q_{n}^{K_{n}}=2 D_{q_{n}^{2}} q_{n}^{q_{n}^{2}} .
$$

Then, $d_{n}>q_{n}^{K_{n}}=e^{q_{n}^{2} \log q_{n}}$, and hence

$$
q_{n}^{2} \log q_{n}<\log d_{n} .
$$

Also, as in (4), from (7) it follows that

$$
K_{n}>\frac{\log d_{n}}{\log \left(2.8 q_{n}\right)}
$$

for each sufficiently large $n$.

Set

$$
R_{n}:=1+\frac{\log \left(3 q_{n}\right) \log \log d_{n}}{\log d_{n}}
$$


Evidently, in view of (6) the disc with center at $\xi$ and radius $R_{n}+1 / q_{n}^{2}$ covers the disc with center at $p_{n} / q_{n}$ and radius $R_{n}$. By Theorem 2, the latter disc contains a full set of conjugates of an algebraic integer of degree $d_{n}$. Thus, by (10), it suffices to show that

$$
\frac{\log \left(3 q_{n}\right) \log \log d_{n}}{\log d_{n}}+\frac{1}{q_{n}^{2}}<\frac{\left(\log \log d_{n}\right)^{2}}{2 \log d_{n}} .
$$

Now, we will verify (11) using (8) and (9). Evidently, $q_{2}>1$ so for $n \geq 2$ we can write (8) in the form $2 \log q_{n}+\log \log q_{n}<\log \log d_{n}$. Consequently,

$$
2 \log \left(3 q_{n}\right)=\log 9+2 \log q_{n}<\log 9+\log \log d_{n}-\log \log q_{n}<\log \log d_{n}-2
$$

for all sufficiently large $n$. This gives an upper bound for the first term in (11):

$$
\frac{\log \left(3 q_{n}\right) \log \log d_{n}}{\log d_{n}}<\frac{\left(\log \log d_{n}\right)^{2}-2 \log \log d_{n}}{2 \log d_{n}}=\frac{\left(\log \log d_{n}\right)^{2}}{2 \log d_{n}}-\frac{\log \log d_{n}}{\log d_{n}} .
$$

Next, by (9), we find that $\log d_{n}<K_{n} \log q_{n}=q_{n}^{2} \log \left(2.8 q_{n}\right)$. This yields $q_{n}>$ $\sqrt{\log d_{n} / \log \log d_{n}}$ for $n$ large enough. Therefore,

$$
\frac{1}{q_{n}^{2}}<\frac{\log \log d_{n}}{\log d_{n}}
$$

By adding both displayed estimates we obtain (11). This, by (7), completes the proof of the theorem with the set

$$
S:=\left\{2 D_{q_{n}^{2}} q_{n}^{q_{n}^{2}}, n=n_{0}, n_{0}+1, \ldots\right\},
$$

where $n_{0}$ is large enough.

We remark that by using Hurwitz theorem instead of that of Dirichlet (with $1 / q^{2}$ replaced by $1 / \sqrt{5} q^{2}$ in (6)) one gets no advantage, since it implies the same result.

Acknowledgement. This research was funded by the European Social Fund according to the activity Improvement of researchers qualification by implementing world-class R\&D projects of Measure No. 09.3.3-LMT-K-712-01-0037.

\section{References}

[1] Capparelli, S., A. Del Fra, and C. Sciò: On the span of polynomials with integer coefficients. - Math. Comp. 79, 2010, 967-981.

[2] Dubickas, A.: On intervals containing full sets of conjugates of algebraic integers. - Acta Arith. 91, 1999, 379-386.

[3] Dubickas, A.: Totally real algebraic integers in small intervals. - Lith. Math. J. 40, 2000, $236-240$.

[4] Ennola, V.: Conjugate algebraic integers in an interval. - Proc. Amer. Math. Soc. 53, 1975, 259-261.

[5] Ennola, V., and C. J. Smyth: Conjugate algebraic numbers on a circle. - Ann. Acad. Sci. Fenn. Ser. A I Math. 582, 1974, 1-31.

[6] Ennola, V., and C. J. Sмyтh: Conjugate algebraic numbers on circles. - Acta Arith. 29, 1976, 147-157.

[7] Feкete, M.: Über die Verteilung der Wurzeln bei gewissen algebraischen Gleichungen mit ganzzahligen Koeffizienten. - Math. Z. 17, 1923, 228-249.

[8] Fekete, M., and G. Szegö: On algebraic equations with integral coefficients whose roots belong to a given point set. - Math. Z. 63, 1955, 158-172.

[9] Flammang, V., G. Rhin, and Q. Wu: The totally real algebraic integers with diameter less than 4. - Mosc. J. Comb. Number Theory 1, 2011, 17-25. 
[10] Grandcolas, M.: Diameters of complete sets of conjugate algebraic integers of small degree. - Math. Comp. 67, 1998, 821-831.

[11] Grandcolas, M.: Weighted diameters of complete sets of conjugate algebraic integers. - Bull. Austral. Math. Soc. 57, 1998, 25-36.

[12] Grandcolas, M.: Problèmes de Favard généralisés. - Bull. Math. Soc. Sci. Math. Roumanie (N.S.) 57:105, 2014, 403-407.

[13] Kronecker, L.: Zwei Sätze über Gleichungen mit ganzzahligen Coefficienten. - J. Reine Angew. Math. 53, 1857, 173-175.

[14] OdLyzko, A. M.: Lower bounds for discriminants of number fields. II. - Tôhoku Math. J. 29, 1977, 209-216.

[15] Robinson, R. M.: Intervals containing infinitely many sets of conjugate algebraic integers. In: Studies in Mathematical Analysis and Related Topics, Stanford, 1962, 305-315.

[16] Rosser, J. B., and L. Schoenfeld: Approximate formulas for some functions of prime numbers. - Illinois J. Math. 6, 1962, 64-94.

[17] SchuR, I.: Über die Verteilung der Wurzeln bei gewissen algebraischen Gleichungen mit ganzzahligen Koeffizienten. - Math. Z. 1, 1918, 377-402.

[18] Zaïmi, T.: Minoration du diamètre d'un entier algébrique totalement réel. - C. R. Acad. Sci. Paris Sér. I Math. 319, 1994, 417-419.

Received 28 November 2018 • Accepted 9 September 2019 